\title{
MINIMAXPRINZIPE ZUR BESTIMMUNG DER EIGENWERTE $J$-NICHTNEGATIVER OPERATOREN
}

\author{
BJÖRN TEXTORIUS
}

\section{Einleitung.}

In den Arbeiten [5] und [6] haben P. D. Lax und R. S. Phillips Minimaxprinzipe zur Bestimmung der Eigenwerte $J$-nichtnegativer, vollstetiger Operatoren im Hilbertraum bewiesen. Anliegen dieser Note ist die Utbertragung dieser Resultate auf einen allgemeineren Fall. Die Minimaxprinzipe lassen sich u.a. zur Untersuchung des Spektrums des Monodromieoperators gewisser kanonischer Differentialgleichungen im Hilbertraum anwenden (siehe [7]).

Die Arbeit wurde durch Mittel der NFR unterstützt. Der Verfasser ist Professor Dr. Heinz Langer sehr zu Dank verpflichtet für die Unterstützung, die er ihm zuteil werden liess.

\section{Minimaxprinzipe.}

Wir verweisen z.B. auf [3] für eine Darstellung der Grundbegriffe der Theorie der $J$-Räume. Es sei $\mathscr{H}$ ein komplexer Hilbertraum bezüglich des positiv definiten Skalarproduktes $(x, y)(x, y \in \mathscr{H})$. Der beschränkte Operator $J$ sei die Differenz zweier selbstadjungierter komplementärer Projektoren $P_{+}, P_{-}$:

$$
J=P_{+}-P_{-}, \quad P_{+}+P_{-}=I,
$$

wo $P_{+}, P_{-} \neq 0$. Mit Hilfe des Operators $J$ sei ein indefinites Skalarprodukt $[x, y](x, y \in \mathscr{H})$ durch die Beziehung

$$
[x, y]=(J x, y)
$$

erklärt. Den mit diesen beiden Skalarprodukten versehenen Raum $\mathscr{H}$ nennen wir einen $J$-Raum.

Es bezeichne [ $\mathscr{H}$ ] die Menge aller beschränkten linearen Operatoren $A$ mit Definitionsbereich $D(A)=\mathscr{H}$ und Wertebereich $R(A) \subset \mathscr{H}$.

Ein Operator $A$ heisst $J$-nichtnegativ wenn $[A x, x] \geqq 0, x \in D(A)$ gilt.

Eingegangen am 20. Januar, 1974. 
Wir definieren die Mengen $\mathscr{P}_{+}, \mathscr{P}_{-}, \mathscr{P}_{0} \subset \mathscr{H}$ folgendermassen:

$$
\mathscr{P}_{+}=\{x \mid[x, x] \geqq 0\}, \quad \mathscr{P}_{-}=\{x \mid[x, x] \leqq 0\}, \quad \mathscr{P}_{0}=\{x \mid[x, x]=0\},
$$

und bezeichnen durch $\mathscr{M}_{+}$(bzw. $\mathscr{M}_{-}$) die Menge aller maximalen Teilräume aus $\mathscr{P}_{+}$(bzw. $\left.\mathscr{P}_{-}\right)$.

Es sei $A$ ein $J$-selbstadjungierter und $J$-nichtnegativer Operator aus $[\mathscr{H}]$,

$$
\mathscr{N}_{A}=\{x \mid x \in \mathscr{H},[A x, x]=[x, x]=0\} .
$$

Für $x \in \mathscr{N}_{A}$ setzen wir $[A x, x][x, x]^{-1}=0$. Aus den Eigenschaften der Spektralfunktion des Operators $A$ ([2]) folgt, dass

$$
[\max \sigma(A)][\min \sigma(A)] \leqq 0
$$

gilt, wobei $\sigma(A)$ das Spektrum von $A$ bezeichnet. Wir führen auch die Bezeichnung $\sigma_{p}(A)$ für das Punktspektrum ein.

SATz 1. Es gilt

$$
\begin{aligned}
\max \sigma(A) & =\inf _{\mathscr{L}_{\in \mathscr{M}+}} \sup _{x \in \mathscr{L}}[A x, x] /[x, x] \\
\min \sigma(A) & =\sup _{\mathscr{L}_{\mathscr{M}_{-}}} \inf _{x \in \mathscr{L}}[A x, x] /[x, x]
\end{aligned}
$$

Beweis. Wir führen den Beweis nur für den Fall $\max \sigma(A)$ durch. Die Beziehung für $\min \sigma(A)$ folgt durch eine ähnliche Überlegung. Bekanntlich (siehe [4]) gibt es einen maximalen $J$-nichtnegativen Teilraum $\mathscr{L}_{+}$max derart, dass

$$
A \mathscr{L}_{+}{ }^{\max } \subset \mathscr{L}_{+}{ }^{\max }, \quad \sigma\left(A \mid \mathscr{L}_{+}{ }^{\max }\right) \subset[0, \infty)
$$

gilt. Es sei $\mathscr{L}_{0}$ der isotrope Teilraum von $\mathscr{L}_{+}{ }^{\max }$, d.h.

$$
\mathscr{L}_{0}=\left\{x \mid x \in \mathscr{L}_{+}{ }^{\max },\left[x, \mathscr{L}_{+}{ }^{\text {max }}\right]=0\right\} .
$$

Offensichtlich gilt $\mathscr{L}_{0}=\mathscr{N}_{A} \cap \mathscr{L}_{+}{ }^{\max }$. Wir zeigen zuerst

$$
\max \sigma(A) \geqq \sup _{x \in \mathscr{L}_{+}}{ }^{\max }\{[A x, x] /[x, x]\} .
$$

Es sei $A_{+}=A \mid \mathscr{L}_{+}{ }^{\text {max }}$. Durch eine von M. G. Krejn stammende Überlegung folgt wie in [6] für $x \notin \mathscr{L}_{0}$ die Beziehung

$$
\left[A_{+} x, x\right] /[x, x] \leqq r_{\sigma}\left(A_{+}\right)=\max \sigma(A) .
$$

Für $x \in \mathscr{L}_{0}$ ist diese Ungleichung definitionsgemäss erfüllt. Folglich gilt

$$
\begin{aligned}
\max \sigma(A) & \geqq \sup _{x \in \mathscr{L}_{+}}{ }^{\max }\{[A x, x] /[x, x]\} \\
& \geqq \inf _{\mathscr{L}_{\mathcal{A}_{+}}{ }^{+} \sup _{x \in \mathscr{L}}[A x, x] /[x, x] \geqq 0 .}
\end{aligned}
$$


Falls $\max \sigma(A)=0$ ist, bleibt nichts zu beweisen. Falls $\max \sigma(A)>0$ ist, verfahren wir folgendermassen. Es sei $\mathscr{L} \in \mathscr{M}_{+}$beliebig,

$$
\Delta=[\max \sigma(A)-\eta, \max \sigma(A)] \quad(\max \sigma(A)>\eta>0)
$$

und $A(\Delta)=A E(\Delta)$, wo $E$ die Spektralfunktion von $A$ ist. Auf Grund der Zerlegung von $A$ bezüglich der Spektralfunktion gilt

$$
[A(\Delta) x, x] /[x, x] \leqq[A x, x] /[x, x], \quad x \in \mathscr{L} .
$$

Für $x \in \mathscr{L} \cap \mathscr{N}_{A}$ steht auf der rechten Seite 0 . Aus der Beziehung $[A x, x] \geqq$ $[A(\Delta) x, x] \geqq 0$ folgt $\mathscr{N}_{A} \subset \mathscr{N}_{A(\Delta)}$, und daher steht auch auf der linken Seite definitionsgemäss 0 .

Bekanntlich gibt es ein maximales duales $\operatorname{Paar}\{\mathscr{N}, \mathscr{P}\}$ von Teilräumen, so dass $E(\Delta) \mathscr{H}$ in $\mathscr{P}$ enthalten ist, $\mathscr{N}$ (bzw. $\mathscr{P}$ ) gleichmässig $J$-negativ (bzw. $J$-positiv) ist und $\mathscr{H}=\mathscr{N}+\mathscr{P}$ gilt, wobei + die direkte und + die $J$-orthogonale Summe bezeichnet. Es sei $K_{\mathscr{L}}$ der Winkeloperator von $\mathscr{L} \in \mathscr{M}_{+}$bezüglich diser Zerlegung, folglich besteht für jedes $x \in \mathscr{L}$ die Beziehung

$$
x=x_{+}+K_{\mathscr{L}} x_{+}, \quad x_{+} \in \mathscr{P} .
$$

Offensichtlich gilt $E(\Delta) \mathscr{H} \subset \mathscr{P} \subset \mathscr{H}$ und daher $E(\Delta) \mathscr{P}=E(\Delta) \mathscr{H}$. Es folgt $A(\Delta) \mathscr{P} \subset \mathscr{P}$ und auch $A(\Delta) \mathscr{N}=\{0\}$.

Das Skalarprodukt $[\cdot, \cdot]$ erzeugt auf $\mathscr{P}$ eine Metrik, die mit der ursprünglichen äquivalent ist. Somit gilt

$$
\begin{aligned}
\max \sigma(A) & =\sup _{x_{+} \in \mathscr{P}}\left[A(\Delta) x_{+}, x_{+}\right] /\left[x_{+}, x_{+}\right] \\
& \leqq \sup _{x_{+} \in \mathscr{P}} \frac{\left[A ( \Delta ) \left(x_{+}+K_{\left.\left.\mathscr{L} x_{+}\right), x_{+}+K_{\mathscr{L}} x_{+}\right]}\right.\right.}{\left[x_{+}, x_{+}\right]+\left[K_{\mathscr{L}} x_{+}, K_{\mathscr{L}} x_{+}\right]} \\
& =\sup _{x \in \mathscr{L}}[A(\Delta) x, x] /[x, x] .
\end{aligned}
$$

Aus den Beziehungen (2) und (3) folgt $\max \sigma(A) \leqq \sup _{x \in \mathscr{L}}[A x, x] /[x, x]$. Daraus ergibt sich

$$
\max \sigma(A) \leqq \inf _{\mathscr{L} \in \mathscr{M}_{+}} \sup _{x \in \mathscr{L}}[A x, x] /[x, x] .
$$

Die Beziehungen (1) und (4) beweisen die Behauptung.

Bemerkung. Im Falle $0 \notin \sigma_{p}(A)$ ist $\mathscr{N}_{A}=\{0\}$. Sind die von Phillips getroffenen Kompaktheitsvoraussetzungen erfüllt, so besteht der Unterschied zwischen Satz 1 und dem entsprechenden Ergebnis aus [6] darin, dass hier alle $\mathscr{L} \in \mathscr{M}_{+}$betrachtet werden, während Phillips nur die streng $J$-positiven Teilräume aus $\mathscr{M}_{+}$benutzt. 
Folaterung. Besteht für jedes $x \in \mathscr{P}_{+}$(bzw. jedes $\left.x \in \mathscr{P}_{-}\right)$die Beziehung $\left[A_{1} x, x\right] \geqq\left[A_{0} x, x\right] \geqq 0\left(A_{0}, A_{1} \in[\mathscr{H}]\right)$, dann gilt

$$
\max \sigma\left(A_{1}\right) \geqq \max \sigma\left(A_{0}\right), \quad \min \sigma\left(A_{1}\right) \leqq \min \sigma\left(A_{0}\right) .
$$

BEWEIS.

$$
\begin{aligned}
& \max \sigma\left(A_{0}\right)=\inf _{\mathscr{L} \in \mathscr{M}_{+}} \sup _{x \in \mathscr{L}}\left[A_{0} x, x\right] /[x, x] \\
& \leqq \inf _{\mathscr{L} \in \mathscr{M}_{+}} \sup _{x \in \mathscr{L}}\left[A_{1} x, x\right] /[x, x]=\max \sigma\left(A_{1}\right), \\
& \min \sigma\left(A_{0}\right)=\sup _{\mathscr{L}_{\epsilon_{\mathscr{N}}}} \inf _{x \in \mathscr{L}}\left[A_{0} x, x\right] /[x, x] \\
& \geqq \sup _{\mathscr{L} \in \mathscr{M}-} \inf _{x \in \mathscr{L}}\left[A_{1} x, x\right] /[x, x]=\min \sigma\left(A_{1}\right) .
\end{aligned}
$$

Unter dem wesentlichen Spektrum $\sigma_{w}(A)$ des Operators $A$ verstehen wir die Vereinigung von dem stetigen Spektrum mit den Punkten unendlicher Vielfachheit sowie den nicht isolierten Punkten aus dem Punktspektrum. Wir setzen noch $\alpha_{+}=\sup \sigma_{w}(A)$.

LeMma 1. Ist $\operatorname{dim} P_{+} \mathscr{H}=\infty$, so gilt $\alpha_{+} \geqq 0$.

BeweIs. Falls $\alpha_{+}<0$ ergibt die Spektralfunktion von $A$ eine Zerlegung $\mathscr{H}=\mathscr{H}_{1}+\mathscr{H}_{2}$ mit einem maximalen $J$-nichtnegativen Teilraum $\mathscr{H}_{2}$, $\operatorname{dim} \mathscr{H}_{2}<\infty$. Gemäss Voraussetzung hat aber jeder maximale $J$-nichtnegative Teilraum unendliche Dimension. Somit gilt $\alpha_{+} \geqq 0$.

Die Voraussetzung in Lemma 1 sei jetzt erfüllt. Wir definieren eine Folge $\left(\lambda_{n}+(A)\right)_{n=1}^{\infty}$ folgendermassen. Besteht $\sigma(A) \cap\left(\alpha_{+}, \infty\right)$ aus unendlich vielen Punkten, so sei $\left(\lambda_{n}+(A)\right)_{n=1}^{\infty}$ die Folge dieser Eigenwerte, nach nichtsteigender Grösse geordnet und jeder so oft gezählt, wie seine Vielfachheit angibt. Enthält $\sigma(A) \cap\left(\alpha_{+}, \infty\right)$ genau $N(0 \leqq N<\infty)$ Eigenwerte, jeder entsprechend seiner Vielfachheit oft gezählt, so seien im Falle $N>0, \lambda_{1}+(A), \ldots \lambda_{N}+(A)$ wie oben definiert, und es sei

$$
\lambda_{N+1}^{+}(A)=\lambda_{N+2}^{+}(A)=\ldots=\alpha_{+} ;
$$

im Falle $N=0$ setzen wir $\lambda_{1}{ }^{+}(A)=\lambda_{2}{ }^{+}(A)=\ldots=\alpha_{+}$.

Entsprechend werde die Folge $\left(\lambda_{n}-(A)\right)_{n=1}^{\infty}$ mit Hilfe von $\alpha_{-}=\inf \sigma_{w}(A)$ definiert. Mit der in Satz 1 benutzten Methode können wir einige Minimaxprinzipe zur Bestimmung der Folgen $\left(\lambda_{n}+(A)\right)_{n=1}^{\infty}$ beweisen.

SATZ 2. Es gilt $\left(n \in Z_{+}\right)$

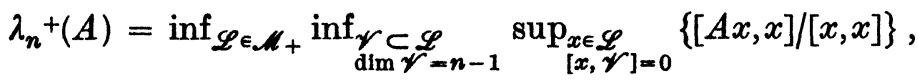

$$
\begin{aligned}
& \lambda_{n}-(A)=\sup _{\mathscr{L} \in \mathscr{M}-} \sup _{\operatorname{dim} \mathscr{\mathscr { V }}=n-1} \inf _{\substack{x \in \mathscr{L} \\
[x, \mathscr{V}]=0}}\{[A x, x] /[x, x]\} .
\end{aligned}
$$


Bewars. Wie in Satz 1 führen wir den Beweis nur für die Folge $\left(\lambda_{n}{ }^{+}(A)\right)_{n=1}^{\infty}$ durch. $\mathscr{L}_{+}{ }^{\max }$ bezeichne dabei denselben maximalen $J$-nichtnegativen Teilraum von $\mathscr{H}$ wie dort. Wir betrachten zuerst den Fall, wo $\sigma(A) \cap\left(\alpha_{+}, \infty\right)$ aus unendlich vielen Punkten besteht, bezeichnen ein Eigenelement, das dem Eigenwert $\lambda_{n}{ }^{+}(A)$ entspricht, mit $\varphi_{n}{ }^{+}\left(n \in \mathbf{Z}_{+}\right)$ und die lineare Hülle von $\varphi_{1}{ }^{+}, \ldots, \varphi_{n}{ }^{+}$mit $\mathscr{V}_{n}$. Die Teilräume $\mathscr{V}_{n}$ sind gleichmässig $J$-positiv, und folglich gilt für jedes $n \in Z_{+}$:

$$
\mathscr{L}_{+}{ }^{\max }=\mathscr{V}_{n-1}+\mathscr{V}_{n-1}^{\prime 3},
$$

wo $\mathscr{V}_{n-1}^{\prime}$ das $J$-orthogonale Komplement von $\mathscr{V}_{n-1}$ in $\mathscr{L}_{+} \max$ bezeichnet. Offensichtlich gilt $A \mathscr{V}_{n-1}=\mathscr{V}_{n-1}$, und da $A J$-selbstadjungiert ist, gilt auch $A \mathscr{V}_{n-1}^{\prime} \subset \mathscr{V}_{n-1}^{\prime \prime}$. Es sei $A_{n-1}=A \mid \mathscr{V}_{n-1}^{\prime}$. Für $x \in \mathscr{V}_{n-1}^{\prime}$ folgt wie oben

$$
\left[A_{n-1} x, x\right] \leqq r_{\sigma}\left(A_{n-1}\right)[x, x],
$$

und da $r_{\sigma}\left(A_{n-1}\right)=\lambda_{n}+(A)$ gilt, ist die Ungleichung

$$
\left[A_{n-1} x, x\right] /[x, x] \leqq \lambda_{n}+(A)
$$

für $x \notin \mathscr{L}_{0}$ erfüllt. Für $x \in \mathscr{L}_{\mathbf{0}}$ gilt dieselbe Ungleichung definitionsgemäss. Somit ergibt sich

$$
0 \leqq \mu_{n}{ }^{+}(A) \leqq \lambda_{n}+(A),
$$

wobei

definiert ist.

$$
\mu_{n}+(A)=\inf _{\mathscr{L} \in \mathscr{M}_{+}} \inf _{\mathscr{d i m} \mathscr{\mathscr { V }}=n-1} \sup _{\substack{x \in \mathscr{V} \\[x, \mathscr{V}]=0}}\{[A x, x] /[x, x]\}
$$

Wir betrachten jetzt den Fall, wo $\sigma(A) \cap\left(\alpha_{+}, \infty\right)$ aus höchstens endlich vielen Punkten besteht. Ist dabei $\alpha_{+}>0$, so lässt sich der obige Beweis auch auf diesen Fall mit Hilfe der Spektralfunktion von $A$ übertragen. Der Fall $\alpha_{+}=0, N=0$ ist in Satz 1 behandelt. Nur der Fall $\alpha_{+}=0, N>0$ bleibt zu behandeln. Die Behauptung für $\lambda_{1}+(A), \ldots, \lambda_{N+1}^{+}(A)$ ergibt sich aus der obigen Überlegung. Da weiter die Folge $\left(\mu_{n}+(A)\right)_{n=1}^{\infty}$ nichtnegativ und fallend ist, folgt die Behauptung.

Es sei $\mathscr{L} \in \mathscr{M}_{+}$beliebig. Um die umgekehrte Ungleichung für alle $\lambda_{n}{ }^{+}(A)>0 \mathrm{zu}$ beweisen, wählen wir Intervalle

$$
\Delta_{n}=\left[\lambda_{n}+(A)-\delta, \lambda_{1}+(A)\right], \quad \delta>0,
$$

mit der Eigenschift, dass $\lambda_{m}{ }^{+}(A) \notin \Delta_{n}$ gilt, falls $m>n$ und $\lambda_{m}{ }^{+}(A)<\lambda_{n}{ }^{+}(A)$, und setzen $A\left(\Delta_{n}\right)=A E\left(\Delta_{n}\right)$. Es gibt ein maximales duales Paar $\left\{\mathscr{N}_{n}, \mathscr{P}_{n}\right\}$ von Teilräumen mit den in Satz 1 angegebenen Eigenschaften. Offensichtlich entspricht jeder $(n-1)$-dimensionale Teilraum $\mathscr{V}$ von $\mathscr{L}$ einem $(n-1)$-dimensionalen Teilraum $\mathscr{V}^{\prime}$ von $\mathscr{P}_{n}$. Auf Grund des wohlbekannten Minimaxprinzips gilt 


$$
\lambda_{n}+(A)=\inf _{\mathscr{V}^{\prime} \mathscr{V}^{\prime}=n-1} \sup _{\substack{x_{+} \in \mathscr{P}^{n} \\\left[x, \mathscr{V}^{\prime}\right]=0}}\left\{\left[A\left(\Delta_{n}\right) x_{+}, x_{+}\right] /\left[x_{+}, x_{+}\right]\right\},
$$

und daraus folgt (vgl. (3))

$$
\lambda_{n}+(A) \leqq \inf _{\mathscr{d i m} \mathscr{V}=n-1} \sup _{[x \in \mathscr{L}]=0}\left(\left[A\left(\Delta_{n}\right) x, x\right] /[x, x]\right) .
$$

Durch die Beziehung (6) und die Abschätzung

$$
\left[A\left(\Delta_{n}\right) x, x\right] /[x, x] \leqq[A x, x] /[x, x], \quad x \in \mathscr{L},
$$

ergibt sich die Behauptung. Der Satz ist somit bewiesen.

Folgerung. Besteht für jedes $x \in \mathscr{P}_{+}$(bzw. jedes $x \in \mathscr{P}_{-}$) die Beziehung

$$
\left[A_{1} x, x\right] \geqq\left[A_{0} x, x\right] \geqq 0, \quad\left(A_{0}, A_{1} \in[\mathscr{H}]\right),
$$

dann gelten die Abschätzungen

$$
\lambda_{n}{ }^{+}\left(A_{1}\right) \geqq \lambda_{n}{ }^{+}\left(A_{0}\right), \quad\left(\text { bzw. } \lambda_{n}{ }^{-}\left(A_{1}\right) \leqq \lambda_{n}-\left(A_{0}\right)\right) \quad\left(n \in \mathbf{Z}_{+}\right) .
$$

Wir bezeichnen die Menge der approximativen Eigenwerte des Operators $A \in[\mathscr{H}]$ mit $\sigma_{0}(A)$. Jeder Randpunkt von $\sigma(A)$ gehört zu $\sigma_{0}(A)$.

Die Beziehung

$$
\sigma_{0}\left(A_{1} A_{2}\right) \backslash\{0\}=\sigma_{0}\left(A_{2} A_{1}\right) \backslash\{0\} \quad\left(A_{1}, A_{2} \in[\mathscr{H}]\right)
$$

ist leicht verifizierbar und gilt auch für die Spektra, falls diese reell sind. Durch eine einfache Rechnung ergibt sich daraus für einen $J$-nichtnegativen und folglich $J$-selbstadjungierten Operator $A$ die Beziehung

$$
\sigma(A)=\sigma\left((A J)^{\frac{1}{t}} J(A J)^{\frac{1}{\mathfrak{b}}}\right) .
$$

Gehört $\lambda=0$ nicht zum Punktspektrum des Operators $A$, so haben offensichtlich die Eigenwerte dieselbe Vielfachheit bezüglich $A$ wie bezüglich $(A J)^{\ddagger} J(A J)^{\ddagger}$. Das in der Arbeit [5] angegebene Minimaxprinzip lässt sich jetzt verallgemeinern.

Satz 3. Der Operator $A \in[\mathscr{H}]$ sei $J$-nichtnegativ, $0 \notin \sigma_{p}(A)$. Dann gilt

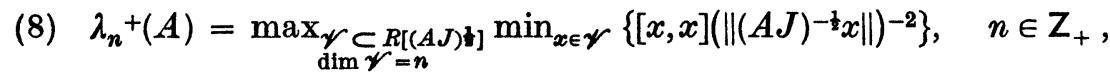

$$
\lambda_{n}-(A)=\min _{\mathscr{d} \mathcal{V} \subset \mathscr{V}=n} \max _{x \in \mathscr{V}}\left\{[x, x]\left(\left\|(A J)^{-\mathbf{1}} x\right\|\right)^{-2}\right\}, \quad n \in \mathrm{Z}_{+} .
$$


BeweIs. Es gilt

$$
\begin{aligned}
& \sup _{x \in R\left[(A J)^{\frac{1}{1}}\right]}\left\{[x, x]\left(\left\|(A J)^{-\frac{1}{2}} x\right\|\right)^{-2}\right\}=\max \sigma\left((A J)^{\frac{1}{1}} J(A J)^{\frac{1}{2}}\right)=\max \sigma(A), \\
& \inf _{x \in R\left[(A J)^{\frac{1}{2}}\right]}\left\{[x, x]\left(\left\|(A J)^{-\frac{1}{2} x}\right\|\right)^{-2}\right\}=\min \sigma\left((A J)^{\frac{1}{2}} J(A J)^{\frac{1}{2}}\right)=\min \sigma(A) .
\end{aligned}
$$

Somit ist (7) bewiesen. Für den Beweis von (8) verweisen wir auf [5] und die obige Bemerkung über die Vielfachheit der Eigenwerte.

Folgerung. Unter den Voraussetzungen des Satzes 3 gilt

$$
\begin{gathered}
\lambda_{n}{ }^{+}(A)=\max _{\mathscr{d i m} \mathscr{\mathscr { V } = n}} \min _{x \in \mathscr{V}}\left\{[x, x] /\left[A^{-1} x, x\right]\right\}, \\
\lambda_{n}-(A)=\min _{\mathscr{d} \subset \mathscr{V} \subset R(A)} \max _{x \in \mathscr{V}}\left\{[x, x] /\left[A^{-1} x, x\right]\right\}, \quad n \in \mathbf{Z}_{+} .
\end{gathered}
$$

BeweIs. Wir beweisen nur die Behauptung für $\lambda_{n}{ }^{+}(A)$. Es gilt $R(A)=$ $R(A J) \subset R\left((A J)^{\frac{1}{2}}\right)$. Somit bestehen die Beziehungen

$$
\begin{aligned}
& \max _{\mathscr{d i m} \mathscr{V}=n} \min _{x \in \mathscr{V}}\left\{[x, x] /\left[A^{-1} x, x\right]\right\} \\
& =\max _{\operatorname{dim} \mathscr{V}=n} \min _{x \in \mathscr{V}(A)}\left\{[x, x]\left(\left\|(A J)^{-\frac{1}{2}} x\right\|\right)^{-2}\right\} \\
& \left.\leqq \max _{\mathscr{d i m} \mathscr{V}=n} \min _{x \in \mathscr{V}}\left\{[x, x]\left(\|(A J)^{\left.\frac{1}{2}\right]}\right)^{-\frac{1}{2}} x \|\right)^{-2}\right\}=\lambda_{n}{ }^{+}(A) .
\end{aligned}
$$

Wir führen den Beweis der umgekehrten Ungleichung nur unter der Voraussetzung, dass $\sigma(A) \cap\left(\alpha_{+}, \infty\right)$ aus endlich vielen Punkten besteht. Die anderen Fälle lassen sich wie im Beweis des Satzes 2 behandeln für $\alpha_{+}>0$ benutzt man dabei wieder die Spektralfunktion. Es seien die Elemente $\psi_{i}\left(i \in \mathbf{Z}_{+}\right)$durch die Beziehungen

$$
(A J)^{\frac{1}{2}} J(A J)^{\frac{1}{2}} \psi_{i}=\lambda_{i}{ }^{+}(A) \psi_{i}
$$

erklärt. Wir bezeichnen die lineare Hülle von $\psi_{1}, \ldots \psi_{n}$ mit $\mathscr{V}_{n}$ und setzen

$$
\mathscr{W}_{n}=(A J)^{\frac{1}{2}} \mathscr{V}_{n}
$$

Offensichtlich gilt $\operatorname{dim} \mathscr{W}_{n}=\operatorname{dim} \mathscr{V}_{n}=n, \mathscr{W}_{n} \subset R(A)$. Es sei weiter

Daraus folgt

$$
\psi_{i}{ }^{\prime}=(A J)^{\frac{1}{3}} \psi_{i} \quad\left(i \in Z_{+}\right) .
$$

$$
A \psi_{i}{ }^{\prime}=(A J)^{\frac{1}{2}}(A J)^{\frac{1}{4}} J(A J)^{\frac{1}{\frac{1}{2}}} \psi_{i}=\lambda_{i}{ }^{+}(A) \psi_{i}{ }^{\prime},
$$

und wir können ohne Beschränkung der Allgemeinheit voraussetzen, dass

$$
\left[\psi_{i}{ }^{\prime}, \psi_{k}{ }^{\prime}\right]=\delta_{i k}, \quad 1 \leqq i, k \leqq n
$$

( $\delta_{i k}=1$ wenn $i=k$, und $\delta_{i k}=0$ sonst) gilt. 
Jedes $x$ in $\mathscr{W}_{n}$ hat die Darstellung $x=\sum_{i=1}^{n} \alpha_{i} \psi_{i}{ }^{\prime}$. Es folgt

$$
\begin{gathered}
\min _{x \in \mathscr{W}_{n}}\left\{\frac{[x, x]}{\left[A^{-1} x, x\right]}\right\}=\min _{\alpha_{i}} \frac{\sum_{i=1}^{n}\left|\alpha_{i}\right|^{2}}{\sum_{i=1}^{n}\left(\lambda_{i}+(A)\right)^{-1}\left|\alpha_{i}\right|^{2}}=\lambda_{n}+(A), \\
\max _{\substack{\operatorname{dim} \mathscr{\mathscr { V }}=n \\
\subset(A)}} \min _{x \in \mathscr{V}}\left\{[x, x] /\left[A^{-1} x, x\right]\right\} \geqq \lambda_{n}+(A) .
\end{gathered}
$$

Die Behauptung ist somit bewiesen.

Wenn man [5, Lemma 4.3] beachtet ergibt sich die Folgerung zum Satz 2 auch aus Satz 3.

Der Operator $A \in[\mathscr{H}]$ sei $J$-nichtnegativ. Wir führen die Bezeichnungen

ein.

$$
\sigma_{1}(A)=\sigma(A) \cap[0, \infty), \quad \sigma_{2}(A)=\sigma(A) \cap(-\infty, 0]
$$

Behauptung 1. Gilt für alle $x \in \mathscr{H}$

so gilt

$$
\begin{gathered}
{\left[A_{1} x, x\right] \geqq\left[A_{0} x, x\right] \geqq 0 \quad\left(A_{0}, A_{1} \in[\mathscr{H}]\right),} \\
\min \sigma_{1}\left(A_{0}\right)>0, \quad \max \sigma_{2}\left(A_{0}\right)<0,
\end{gathered}
$$

und

$$
\max \sigma\left(A_{1}\right) \geqq \max \sigma\left(A_{0}\right), \quad \min \sigma_{1}\left(A_{1}\right) \geqq \min \sigma_{1}\left(A_{0}\right)
$$

$$
\min \sigma\left(A_{1}\right) \leqq \min \sigma\left(A_{0}\right), \quad \max \sigma_{2}\left(A_{1}\right) \leqq \max \sigma_{2}\left(A_{0}\right) \text {. }
$$

BEwEIs. Gemäss Voraussetzung ist $\Delta=\left(\max \sigma_{2}\left(A_{0}\right), \min \sigma_{1}\left(A_{0}\right)\right)$ nicht leer. Es folgt somit

$$
\left[\left(A_{0}-\alpha I\right) x, x\right] \geqq 0, \quad x \in \mathscr{H}, \alpha \in \Delta,
$$

(gemäss [2]) und daher hat auch der Operator $A_{1}$ dieselbe Eigenschaft. Folglich (siehe [2]) gehört $\Delta$ zur Resolventenmenge von $A_{1}$, d.h. est gilt

$$
\min \sigma_{1}\left(A_{1}\right) \geqq \min \sigma_{1}\left(A_{0}\right), \quad \max \sigma_{2}\left(A_{1}\right) \leqq \max \sigma_{2}\left(A_{0}\right) .
$$

Die übrigen Aussagen sind bereits in den Folgerungen zu Satz 1 enthalten.

Wir sind jetzt imstande, die Lage der Spektren der Operatoren $A$ und $J A$ zu vergleichen.

Behauptung 2. Ist der Operator $A \in\left[\mathscr{H}^{]}\right] J$-nichtnegativ, so gilt

$$
-\max \sigma(J A) \leqq \min \sigma(A) \leqq \max \sigma(A) \leqq \max \sigma(J A) .
$$


Bewhis. Es gilt $\sigma(A)=\sigma\left[(J A)^{\frac{1}{}} J(J A)^{\frac{1}{2}}\right]$. Somit bestehen die Beziehungen

$$
\begin{aligned}
\max \sigma(A) & =\max \sigma\left[(J A)^{\frac{1}{2}} J(J A)^{\frac{1}{2}}\right]=\sup _{\|x\|=1}\left((J A)^{\frac{1}{2}} J(J A)^{\frac{1}{2}} x, x\right) \\
& =\sup _{\|x\|=1}\left[(J A)^{\frac{1}{2}} x,(J A)^{\frac{1}{2}} x\right] \leqq \sup _{\|x\|=1}\left\|(J A)^{\frac{1}{2}} x\right\|^{2} \\
& =\sup _{\|x\|=1}(J A x, x)=\max \sigma(J A) . \\
\min \sigma(A) & =\min \sigma\left[(J A)^{\frac{1}{2}} J(J A)^{\frac{1}{2}}\right]=\inf _{\|x\|=1}\left((J A)^{\frac{1}{2}} J(J A)^{\frac{1}{2}} x, x\right) \\
& =\inf _{\|x\|=1}\left[(J A)^{\frac{1}{2}} x,(J A)^{\frac{1}{2}} x\right] \geqq \inf _{\|x\|=1}\left(-\left\|(J A)^{\frac{1}{2}} x\right\|^{2}\right) \\
& =-\max \sigma(J A) .
\end{aligned}
$$

Die Folge $\left(\lambda_{n}(J A)\right)_{n=1}^{\infty}$ ist analog der Folge $\left(\lambda_{n}{ }^{+}(A)\right)_{n=1}^{\infty}$ erklärt.

Behauptung 3. Ist der Operator $A \in[\mathscr{H}] J$-nichtnegativ, so gilt $\left(n \in \mathrm{Z}_{+}\right)$

$$
\lambda_{n}{ }^{+}(A) \leqq \lambda_{n}(J A), \quad \lambda_{n}{ }^{-}(A) \geqq-\lambda_{n}(J A) .
$$

Bewsis. Die Behauptung folgt aus der vorigen Ưberlegung und den Beziehungen

$$
\begin{aligned}
& \lambda_{n}{ }^{+}\left((J A)^{\frac{1}{2}} J(J A)^{\frac{1}{2}}\right)=\max _{\mathscr{d i m} \mathscr{L}} \min _{x \in \mathscr{L}}\left((J A)^{\frac{1}{2}} J(J A)^{\frac{1}{2}} x, x\right), \\
& \lambda_{n}-\left((J A)^{\frac{1}{2}} J(J A)^{\frac{1}{2}}\right)=\min _{\operatorname{dim} \mathscr{L}} \min _{\| x} \max _{\substack{x \in \mathscr{L} \\
\|x\|=1}}\left((J A)^{\frac{1}{2}} J(J A)^{\frac{1}{2}} x, x\right) .
\end{aligned}
$$

Gilt $\left[A_{1} x, x\right] \geqq\left[A_{0} x, x\right] \geqq 0, x \in \mathscr{H}\left(A_{0}, A_{1} \in[\mathscr{H}]\right)$, und $\operatorname{dim} R\left(A_{1}-A_{0}\right)=q<\infty$, so folgt somit aus Satz 2 und Behauptung 3 mit Rücksicht auf wohlbekannte Ergebnisse über endlichdimensionale Störungen

$$
\begin{aligned}
\lambda_{n+q}\left(J A_{0}\right) & \leqq \lambda_{n}\left(J A_{1}\right) \leqq \lambda_{n-q}\left(J A_{0}\right), \\
\lambda_{n}{ }^{+}\left(A_{k}\right) & \leqq \lambda_{n}\left(J A_{k}\right), \quad \lambda_{n}-\left(A_{k}\right) \geqq-\lambda_{n}\left(J A_{k}\right) \quad(k=0,1), \\
\lambda_{n}{ }^{-}\left(A_{1}\right) & \leqq \lambda_{n}-\left(A_{0}\right), \quad \lambda_{n}{ }^{+}\left(A_{0}\right) \leqq \lambda_{n}{ }^{+}\left(A_{1}\right) .
\end{aligned}
$$

Folgerung. Der Operator $A \in[\mathscr{H}]$ sei $J$-nichtnegativ, $0 \notin \sigma_{p}(A)$. Gilt weiter $\operatorname{dim} \mathscr{H}=N<\infty, \operatorname{dim} R\left(P_{-}\right)=\varkappa$, so besteht das Spektrum von $A$ aus $\varkappa$ negativen und $N-\varkappa$ positiven Eigenwerten, und es bestehen die Beziehungen

$$
\begin{aligned}
& \lambda_{x+n}(J A) \leqq \lambda_{n}{ }^{+}(A) \leqq \lambda_{n}(J A), \quad n=1,2, \ldots, N-\varkappa, \\
& -\lambda_{n}(J A) \leqq \lambda_{n}-(A) \leqq-\lambda_{N-\varkappa+n}(J A), \quad n=1,2, \ldots, \varkappa \text {. }
\end{aligned}
$$

Bew eIs. Das negative Spektrum des Operators $A$ besteht bekanntlich aus $x$ negativen Eigenwerten, jeder Eigenwert seiner Vielfachheit ent- 
sprechend oft gezählt. Wir benutzen Behauptung 3 und die Beziehung $\sigma\left[(J A)^{-1}\right]=\sigma\left(A^{-1} J\right)=\sigma\left(J A^{-1}\right)$, um die Abschätzung

$$
\left[\lambda_{N-x-n+1}^{+}(A)\right]^{-1}=\lambda_{n}{ }^{+}\left(A^{-1}\right) \leqq \lambda_{n}\left(J A^{-1}\right)=\left[\lambda_{N-n+1}(J A)\right]^{-1},
$$

$n=1,2, \ldots, N-\varkappa$ zu erhalten. Eine entsprechende Abschätzung gilt für die negativen Eigenwerte.

\title{
LITERATUR
}

1. M. G. Krejn, Uber lineare vollstetige Operatoren in Funktionalräumen mit zwei Normen (ukrainisch), Sbirnik Praz. Inst. Mat. Akad. Nauk URSR 9 (1947), 104-129.

2. M. G. Krejn und Ju. L. Smuljan, J-polare Darstellung von Plus-Operatoren, (russisch), Mat. Issled. 1 (1966), 172-210. (Englische Utbersetzung in Amer. Math. Soc. Transl. (2) 85 (1969), 115-143.)

3. H. Langer, Zur Spektraltheorie J-selbstadjungierter Operatoren, Math. Ann. 146 (1962), 60-85.

4. H. Langer, Invariante Teilräume definisierbarer $J$-selbstadjungierter Operatoren, Ann. Acad. Sci. Fenn. Ser. A I, Mathematica, No 475, Helsinki, 1971.

5. P. D. Lax and R. S. Phillips, Decaying Modes for the Wave Equation in the Exterior of an Obstacle, Comm. Pure Appl. Math. 22 (1969), 737-787.

6. R. S. Phillips, A minimax characterization for the eigenvalues of a positive symmetric operator in a space with an indefinite metric, J. Fac. Sci. Univ. Tokyo Sect. I A Math. 17 (1970), 51-59.

7. B. Textorius, Uber Störungen kanonischer Differentialgleichungen mit operatorwertigen Koeffizienten und definisierbarem Monodromieoperator, erscheint in Math. Z.

\author{
MATHEMATISCHES INSTITUT DER \\ UNIVERSITATT LINK ÖPING \\ 8-581 83 LINK ØPING \\ SCHWEDEN
}

